\title{
RELAÇÕES ENTRE PROFISSIONAIS DE SAÚDE E USUÁRIOS DURANTE AS PRÁTICAS EM SAÚDE
}

\author{
RELATIONSHIPS BETWEEN HEALTH PROFESSIONALS AND USERS THROUGHOUT \\ HEALTH CARE PRACTICES
}

\author{
Maria Denise Schimith ${ }^{1}$ \\ Bruna Sodré Simon ${ }^{2}$ \\ Ana Cristina Passarella Brêtas ${ }^{3}$ \\ Maria de Lourdes Denardin Budó ${ }^{4}$
}

Resumo Apresenta-se uma revisão integrativa sobre estudos que abordam as relações entre profissionais de saúde e usuários durante as práticas em saúde. Objetivou-se identificar os aspectos pesquisados no cotidiano dos serviços acerca dessas relações. A coleta foi realizada nas bases Lilacs e Pubmed segundo os descritores: acolhimento; relações profissional-família; relações profissional-paciente; humanização da assistência; e a palavra 'vínculo' associada ao descritor Sistema Único de Saúde. Selecionaram-se 290 estudos publicados entre 1990 e 2010. Por meio da análise temática, foram criados cinco núcleos de sentido: a relevância da confiança na relação profissionalusuário; sentimentos e sentidos na prática do cuidado; a importância da comunicação nos serviços de saúde; modo de organização das práticas em saúde; e (des)colonialismo. Identificou-se que as relações estabelecidas nas práticas de saúde têm uma dimensão transformadora. No entanto, permanece o desafio de humanizar os serviços de saúde. A enfermagem se destaca na produção do conhecimento nessa temática. Palavras-chave enfermagem; acolhimento; humanização da assistência; Sistema Único de Saúde.
Abstract This article presents an integrative review about studies that address the relationships between health professionals and users in health care practices. It aimed to identify aspects that were researched on the daily life of the services concerning such relationships. Data were collected from the Lilacs and Pubmed databases based on these descriptors: user embracement; professional-family relations; professional-patient relations; humanization of the care; and the bonding word associated to the Single Health System descriptor. Two hundred and ninety studies, published from 1990 to 2010, were selected. Through thematic analyses, five meaning cores were created: the relevance of the confidence in the professionaluser relationship; feelings and senses in the health care practice; the importance of communications in health care services; ways to organize health care practices and (de)colonialism. It was found that relationships established in health care practices have a transformative dimension. However, the challenge to humanize health care services remains. Nursing stands out in the production of knowledge on such theme.

Keywords nursing; user embracement; humanization of assistance; Single Health System. 


\section{Introdução}

As relações estabelecidas entre profissionais de saúde e usuários estão entre os temas desafiadores para a reorganização dos serviços de saúde, portanto, para implementações no Sistema Único de Saúde (SUS).

A experiência na docência e também na assistência permite afirmar que há diversidade na maneira como o usuário é recebido e acolhido. Mesmo em serviços em que o acolhimento está implantado, mantém-se a dificuldade de acesso nos diversos cenários de atenção à saúde. Observa-se, com tal situação, que a organização das equipes de saúde não superou o desafio de se solidarizar pela necessidade dos usuários, não atendendo assim ao princípio da integralidade da atenção.

Para o atendimento ser integral, o encontro com o usuário deve ser guiado pela capacidade do profissional de compreender o sofrimento que se manifesta e o significado mais imediato de suas ações e palavras; no contexto desse encontro concreto, "deixar de vigiar e controlar, para dar lugar à emancipação, à maior autonomia por parte dos sujeitos que sofrem ou que podem vir a sofrer" (Mattos, 2008, p. 348). Portanto, está-se falando de singularidade no atendimento a cada caso. O usuário, ao se sentir singularizado, desfragmentado (Pinheiro e Mattos, 2006), apresenta melhora do quadro clínico, ao mesmo tempo que se observa o aumento de satisfação, tanto do usuário quanto do profissional.

Assim, este estudo 5 torna-se relevante na perspectiva de conhecer a produção do conhecimento, guiado pela seguinte questão de pesquisa: como entender as relações que se estabelecem nas práticas em saúde, considerando a complexidade da saúde, na vida dos sujeitos? Para tanto, investigou-se a produção acadêmica relativa à temática, com o objetivo de identificar os aspectos pesquisados no cotidiano dos serviços acerca das relações entre profissionais de saúde e usuários, conhecendo o estado da arte dessa temática.

\section{Metodologia}

Trata-se de uma revisão integrativa que possibilita ao pesquisador analisar e sintetizar uma determinada temática; também serve para fomentar novas alternativas e apontar lacunas referentes a ela (Mendes, Silveira e Galvão, 2008).

A elaboração da pesquisa integrativa demanda rigor metodológico, segundo Pompeo, Rossi e Galvão (2009). Para atingir tal rigor, foram atendidas as seguintes etapas: elaboração da questão de pesquisa, formulação dos critérios de inclusão e exclusão, escolha dos dados nos estudos selecionados e posterior elaboração das categorias, análise dos dados, inter- 
pretação dos resultados, e por último a apresentação da pesquisa (Mendes, Silveira e Galvão, 2008).

A coleta de dados foi de acordo com os descritores: acolhimento; relações profissional-família; relações profissional-paciente e humanização da assistência; e a palavra 'vínculo' associada ao descritor Sistema Único de Saúde, bem como seus correspondentes em inglês. A busca foi realizada nas bases de dados Lilacs e Pubmed, com formulário avançado, nos meses de maio e junho de 2010, utilizando como limites a pesquisa em humanos e ano de publicação. Foram selecionados artigos e teses publicados entre os anos de 1990 e 2010. A definição do recorte temporal justifica-se por ser o ano de publicação da Lei Orgânica da Saúde (lei n. 8.080/90), a qual regulamenta o SUS.

Foram encontrados, na base de dados Lilacs, 936 artigos e 113 teses e dissertações. Na base Pubmed, encontraram-se 430 artigos. O total de resumos localizados foi de 1.366 artigos e 113 teses ou dissertações. Em um primeiro momento foram eliminados os repetidos; posteriormente aplicaram-se os critérios de inclusão e exclusão. Os critérios de inclusão foram: artigos com resumos disponíveis online, resultados de pesquisas, relatos de experiências e reflexões, teses e dissertações. Já os critérios de exclusão foram: estudos que envolvem o lúdico, necessidades especiais, morte/morrer/cuidado paliativo, serviços privados, aplicativos de Internet e telefone, pesquisa entre acadêmicos ou com objetivo de ensino-aprendizagem e resumos incompletos. Tais critérios justificam-se por se tratar de situações especiais, ou por serem consideradas em serviços especializados. Após a leitura dos resumos, chegou-se a um número final de 290 estudos selecionados.

A organização dos dados para a análise ocorreu com a construção de uma ficha para extração dos dados dos resumos, contendo as seguintes informações: referências do estudo, objetivo, abordagem metodológica, método, tipo de coleta de dados, área, cenário, sujeitos, resultados e conclusões.

A análise seguiu os passos de análise temática, constituída por três fases: a pré-análise, a exploração do material e o tratamento dos resultados obtidos e interpretação (Minayo, 2008). Utilizou-se o software Atlas.ti 5.0 para auxiliar no agrupamento dos núcleos de sentido. Os aspectos que se destacaram na leitura dos resumos foram: a comunicação verbal e não verbal, o papel da informação e do conhecimento nas relações, os sentimentos de usuários, familiares e profissionais, os sentidos do cuidado, a confiança necessária na relação profissional-usuário, o modo de organizar os serviços de saúde e as concepções que permeiam as relações e práticas de cuidados, ou seja, opressoras ou não. Com base nesses critérios, foram constituídos cinco núcleos de sentido: relevância da confiança na relação profissionalusuário; sentimentos e sentidos na prática do cuidado; importância da comunicação nos serviços de saúde; modo de organização das práticas em saúde; e (des)colonialismo. 


\section{Resultados}

Ao observar o recorte temporal escolhido, identificou-se que nos anos de 1990, 1992, 1995 e 1996 não houve trabalhos selecionados. No período de 1991 a 2000, foram 35 estudos. Entre 2001 e 2005 obtivemos 90 estudos selecionados, e no período de 2006 até 2010, foram 168 trabalhos.

Dentre os 290 resumos, somente em 119 foi possível identificar qual era a abordagem da pesquisa, classificando-se em 111 pesquisas qualitativas, três quantitativas, cinco qualiquantitativas. Além desses, em 27 resumos identificou-se que três eram relatos de experiências, 22 revisões de literaturas e dois estudos de caso.

Quanto ao cenário em que os estudos foram realizados ou que eles abordam, o cenário hospitalar aparece em 116 resumos, destacando-se as Unidades de Terapia Intensiva, com 38 estudos, e a Unidade Pediátrica, com 11 estudos. A Atenção Básica é abordada em 37 resumos; destes, 23 representam a Estratégia Saúde da Família (ESF) e 12 a Unidade Básica de Saúde (UBS). Os ambulatórios aparecem em sete trabalhos, e os serviços de apoio são abordados em 13 estudos, sendo que quatro se referem aos Centros de Apoio Psicossocial (Caps); o domicílio é referenciado em quatro estudos.

Apresentam-se a seguir os núcleos de sentidos identificados durante a análise dos resumos.

\section{Relevância da confiança na relação profissional-usuário}

Os resultados de pesquisas realizadas no Brasil referem-se ao respeito ao usuário, compromisso, responsabilidade, cumplicidade e sensibilidade na relação profissional paciente (Molina et al., 2009; Nery et al., 2009; Jorge et al., 2007; Abdalla e Nichiata, 2008). A confiança aparece ainda na concepção de vínculo por profissionais da saúde, especialmente em estudos realizados na ESF, em que o agente comunitário de saúde (ACS) aparece como a figura que pode ligar a população aos demais atores. Nesse cenário, um estudo que abordou o controle da tuberculose (TB) na ESF identifica aspectos potencializadores e fragilizadores do vínculo (Gomes e Sá, 2009).

Os usuários, de modo geral, ao avaliarem o cuidado prestado, reconhecem quando os profissionais demonstram respeito, atenção, elo de afetividade, confiança e credibilidade. Destaca-se um estudo que assinala a importância do vínculo entre cuidadores e adolescentes abrigados, referindo que essa integração possibilita a ressignificação das histórias de vida dos adolescentes (Araújo e Lobo, 2007).

Ao descrever as ações do enfermeiro na atenção ao pré-natal, uma pesquisa informa que, além da atuação técnica, o papel do enfermeiro é pro- 
mover a interação entre as gestantes e equipe, acolhendo e proporcionando vínculo de confiança e credibilidade por parte das gestantes, com consequente adesão ao pré-natal (Duarte e Andrade, 2006).

$\mathrm{Na}$ Argentina, foi publicada uma pesquisa comprovando a relação entre a qualidade da aliança terapêutica e o destino dos tratamentos, adesão ou abandono (Martínez et al., 1997). Em Cuba, o estudo destaca os antecedentes da relação médico-paciente e o marco conceitual dos fundamentos do Sistema Nacional de Saúde, objetivando reforçar para os profissionais de saúde a necessidade de uma identificação plena com o paciente e seus problemas (Pérez Cicili et al., 2003).

Um estudo publicado nos Estados Unidos identificou que a confiança dos pacientes em seus médicos e o compromisso na relação profissionalpaciente influenciam favoravelmente no comportamento alimentar saudável dos pacientes (Berry et al., 2008). Entre as barreiras para o cuidado de insuficiência cardíaca, em pesquisa realizada com nativos das ilhas do Pacífico nos EUA, foi encontrada a falta de confiança nos cuidados médicos, destacando-se a necessidade de facilitar o relacionamento médico-paciente para melhorar as intervenções (Kaholokula et al., 2008).

Outra pesquisa, com pacientes que realizavam consultas em clínicas oftálmicas, evidenciou que quando os profissionais reconhecem os sujeitos de maneira integral, levando em consideração não apenas aspectos biológicos, mas também as questões de crenças e valores, há benefícios quanto ao estabelecimento de relações respeitosas, além de auxílio na tomada de decisões terapêuticas em conjunto, entre profissionais e pacientes (Magyar-Russell et al., 2008).

\section{Sentimentos e sentidos na prática do cuidado}

Nesse núcleo, foram agrupados estudos que apresentaram em seus resultados sentimentos e sentidos das práticas em saúde, bem como significados da interação entre profissionais, usuários e familiares, revelando a percepção desses atores no processo do cuidado. Também aparecem aqui estudos que evidenciaram a postura profissional e a interação com crenças e valores.

A enfermagem surge como a profissão que mais pesquisa e é pesquisada, em diversos cenários do cuidado, como unidades de terapia intensiva (UTI) adultas e pediátricas, outras unidades hospitalares, ESF e domicílio, por exemplo.

Destacam-se, dentre os sentidos do cuidado apresentados, os que se referem aos modos de atuação dos profissionais, ou seja, a atuação técnica e a atuação de interação. Nesse sentido, as pesquisas apresentam como resultado que a assistência não pode estar centrada apenas no elemento técnico, 
mas principalmente no relacionamento profissional-usuário (Davim et al., 2009; Almeida e Silva, 2008; Woiski e Rocha, 2010; Barra et al., 2005). Ao descrever a opinião de puérperas sobre o acolhimento por profissionais de saúde no alojamento conjunto (AC) de uma maternidade pública de Natal (RN), as relações pessoais surgem como ponto fundamental para a humanização do acolhimento na assistência à saúde (Davim et al., 2009). A melhoria da assistência também está configurada nos valores pessoais, cuidado com compromisso aplicado à prática humanística, conforme Caetano et al. (2007). A cultura de humanização hospitalar deve ser voltada para o ser humano que adoece, não à doença (Backes, Lunardi Filho e Lunardi, 2006). Para os usuários de UTI adulto, a interação e a atenção da enfermagem são mais significativas que os cuidados técnicos (Matsuda, Silva e Tisolin, 2003).

Entre os estudos internacionais que abordam essa diferenciação do cuidado, aponta-se um desenvolvido no Chile, que investigou o cuidado percebido por mulheres em situação de parto. O componente relacional foi indicado pelo uso de palavras suaves, o aporte de informações, a presença do familiar e o apoio e respaldo às mães. Diz que o desenvolvimento de habilidades interpessoais, sem esquecer a permanente atualização do componente técnico do cuidado, deve ser o eixo organizador do cuidado (Bravo, Uribet e Contreras, 2008).

Outro estudo, publicado no México, relacionou a satisfação de usuárias dos serviços de pré-natal com o tratamento recebido durante a consulta e o tempo de espera para recebê-la, mas não com a capacidade de resolução clínica do profissional nem com idade e sexo. O tratamento recebido durante a consulta também foi associado ao nível socioeconômico da usuária, sendo que as mais pobres receberam, proporcionalmente, pior tratamento (Bronfman-Pertzovsky et al., 2003).

Destaca-se também um estudo quantitativo publicado no Chile, no qual $96 \%$ das mulheres entrevistadas consideraram importante a capacidade do médico de escutá-las e explicar-lhes claramente a doença e o tratamento, e a segurança expressa. Classificaram como características ideais dos médicos: em primeiro lugar, a segurança; em segundo, a capacidade de explicar; em terceiro, a capacidade de escuta; e em quarto, a empatia (Pérez et al., 2003). Em uma pesquisa realizada nos EUA, com pacientes com dor crônica, a comunicação empática entre profissionais e pacientes foi considerada crucial, pois a comunicação insensível afeta negativamente o indivíduo, reduz a conformidade com o tratamento e aumenta a utilização dos serviços de saúde (Dewar et al., 2009).

Num estudo que objetivou verificar percepções de mulheres com relação ao primeiro exame de prevenção do câncer de colo uterino e identificar atitudes profissionais favoráveis ao bem-estar das mulheres na realização dele, os sentimentos agruparam-se nas categorias vergonha, medo, tensão, 
desconforto e dor. As atitudes profissionais foram reunidas em diálogo, toque, paciência, acolhimento e aproximação do universo das mulheres (Sousa et al., 2008).

Nesse núcleo de sentido também foi possível identificar algumas concepções de humanização, das quais se podem citar as percebidas por enfermeiros como cuidar do outro como gostaria de ser cuidado e ter a visão integral dos usuários (Beck et al., 2009). O atendimento humanizado no hospital está relacionado com a capacidade de abordar o paciente de forma holística e igualitária (Faquinello, Higarashi e Marcon, 2007). No ambiente hospitalar, a humanização ainda mantém-se centrada na figura pessoacliente, evidenciando a pouca atenção ao cuidado e à humanização do sujeito-trabalhador (Amestoy, Schuwartz e Thofehrn, 2006).

\section{Importância da comunicação nas práticas de cuidado}

Um elevado índice dos estudos analisados refere-se à comunicação como componente integrante do processo de humanização da assistência, destacando que para uma prática humanizada torna-se necessária a valorização da comunicação por parte dos profissionais com os sujeitos e seus familiares (Gotardo e Silva, 2005; Faiman et al., 2003; Santos, 1998).

Ainda no viés da humanização, a comunicação é abordada no sentido de possibilitar a integralidade da atenção à saúde, pois, numa comunicação em que os sujeitos sejam escutados de maneira qualificada e satisfatória, podem interagir e compartilhar suas vivências. Ela possibilita ainda que as condutas sejam pautadas e programadas de acordo com o conhecimento dos aspectos socioeconômicos e culturais (Oliveira, 2002).

Assim, estudos revelam que os usuários dos serviços de saúde buscam profissionais qualificados, comprometidos, preparados para escutá-los e realizar uma comunicação acolhedora, com a valorização dos discursos e que tenha resolutividade para as suas necessidades (Oliveira et al., 2008; Hoyos, Cardona e Correa, 2008).

Outro tema que teve destaque no processo de comunicação foi a adesão ao tratamento. Pesquisas revelam que tanto a interrupção do tratamento como a não adesão estão relacionadas na maior parte das vezes à não compreensão das informações sobre o uso adequado dos medicamentos (Assunção e Ursine, 2008; Ganzella e Zago, 2008). Em um estudo sobre a não adesão, os autores ressaltam a necessidade de uma abordagem holística, ou seja, é preciso que os profissionais da saúde trabalhem de maneira conjunta e visualizem os pacientes de forma integral, identificando as condições sociais, econômicas e culturais dos indivíduos, para auxiliar no processo de adesão ao tratamento (Moreira e Araújo, 2002). 
Uma dissertação de mestrado constatou que existe uma contradição na comunicação interpessoal aprendida durante a fase de graduação e depois, ao exercer a profissão (Lima, 1993). Foi possível confirmar isso com o fazer técnico biologicista priorizado no cotidiano de trabalho, em lugar de uma assistência prestada e centrada no sujeito.

Quando a comunicação tem como sujeitos-alvo os profissionais de saúde, apresenta algumas falhas e enfrenta dificuldades. Estudo revela que um local adequado dentro do ambiente de trabalho, para que os profissionais possam se encontrar a fim de discutir e refletir sobre o cotidiano e compartilhar as angústias e satisfações é um fator importante para a humanização (Yokaichiya et al., 2006). Além disso, o pouco tempo disponível para as reuniões é um fator que dificulta os profissionais de estabelecerem o diálogo com colegas de trabalho, familiares e pacientes (Lima, 1993).

Dentre os estudos analisados, três populações encontraram-se vulneráveis às práticas de saúde fragmentadas: os jovens, as mulheres que sofrem com violência e os homens que compartilham experiências com aborto. $\mathrm{O}$ processo de comunicação com os adolescentes tem como eixo principal os assuntos relacionados com a iniciação sexual e a sexualidade de modo geral (Nascimento e Gomes, 2009). Em trabalho realizado com o público feminino, emergiu a dificuldade de proporcionar acolhimento e detectar os casos de violência contra as mulheres (Borsoi, Brandão e Cavalcanti, 2009). Já com os homens, a pesquisa deu-se em torno daqueles que acompanharam mulheres em situação de aborto (Rodrigues e Hoga, 2006). Os estudos citados evidenciaram a necessidade de profissionais com personalidade voltada para o acolhimento, que fossem sensíveis, demonstrassem envolvimento e comprometimento com os sujeitos, requerendo profissionais com especificidades de assistência e comunicação para cada um deles.

Uma boa comunicação auxilia também na organização dos serviços de saúde. Pesquisas revelam que se a população recebesse informações adequadas acerca da situação em que procurar os serviços de urgência e emergência, menor seria o tempo de espera nos serviços, e com isso os casos que realmente necessitam do atendimento teriam uma disponibilidade melhor da equipe de saúde (Carret, Fassa e Domingues, 2009; Coelho e Jorge, 2009; Oliveira, Costa e Soares, 2007).

A comunicação com os familiares e acompanhantes também foi citada entre os trabalhos selecionados. É preciso uma interação pacientes-profissionais-familiares, em que sejam fornecidas informações referentes aos cuidados específicos, pois assim será possível fortalecer os cuidadores para a alta hospitalar. Além disso, os familiares devem ser informados quanto às transferências que acontecem dentro do hospital. Dessa forma, é possível diminuir a angústia, a ansiedade e as preocupações entre os familiares (Lima e Busin, 2008). 
Uma pesquisa realizada no Reino Unido faz referência aos dois tipos de comunicação, a verbal e a não verbal, realizada durante atendimentos de fisioterapeutas. Dos sujeitos, $52 \%$ conseguiram compreender e participaram do processo de comunicação verbal. No entanto, dentre os sujeitos que não compreenderam a comunicação verbal, $84 \%$ apenas tiveram respostas por olhares e 54\% responderam a estímulos de toque terapêutico (Roberts e Bucksey, 2007).

Como fragilidades encontradas nos estudos selecionados, a maior parte deles afirma que muitos profissionais utilizam um linguajar baseado em termos técnicos e científicos; algumas das informações deveriam ser por escrito, o que nem sempre acontece (Araújo, Rodrigues e Rodrigues, 2008; Queiroz et al., 2007; Victor et al., 2003). Além disso, um estudo ressalta que existe negligência nas informações e também que algumas destas nem sempre são iguais entre os profissionais, o que acarreta uma variedade de informações e com desencontros entre elas (Queiroz et al., 2007).

Uma pesquisa sugere que, no processo de comunicação, os profissionais façam referência aos pacientes pelo nome de cada um, que durante o diálogo haja uma relação de 'olho no olho', e que os profissionais estejam dispostos a se comunicar com termos de fácil entendimento e possibilitem uma escuta sensível e qualificada (Gomes e Vianna, 2008).

É preciso ouvir mais e falar menos, pois assim será possível dar ênfase ao processo de diálogo e reduzir a tendência que os profissionais de saúde têm, em especial os médicos, de realizar monólogos (Roter et al., 2008).

\section{Modo de organização das práticas em saúde}

A criação do Programa de Saúde da Família (PSF) em 1994 e, em 2003, a implantação da Política Nacional de Humanização (PNH) desencadearam modificações no modo de organização dos serviços e da atuação dos profissionais.

Diante dessa realidade, estudos revelam que a implementação do PSF aumentou a demanda dos serviços e acarretou mudanças no modelo de atenção. Isso ocasionou a necessidade de se buscar auxílio em outras áreas do conhecimento, gerando a integração dos saberes nas práticas assistenciais e formando equipes multiprofissionais (Nery et al., 2009; Delfini, Sato e Antoneli, 2009).

O processo de trabalho dos profissionais da enfermagem precisou ser direcionado para um novo posicionamento, ou seja, necessitou ser baseado no processo de humanização, integralidade e na melhora da qualidade de vida dos sujeitos (Bicca e Tavares, 2006). A referida transformação do posicionamento é condizente com a $\mathrm{PNH}$, pois esta tem como princípio a atenção integral, por intermédio do acolhimento e do acesso aos usuários (Nascimento, Tesser e Poli Neto, 2008). 
Dentre os achados da pesquisa nesse núcleo de sentido, há um grande índice de estudos selecionados que fazem referência à Saúde da Criança. Eles revelam que uma das principais mudanças no processo de organização é a presença da família ou de cuidadores com vínculo durante o momento de hospitalização. Esse acompanhamento é enfatizado com uma atenção especial da equipe de enfermagem, pois ela pode apoderar-se desse momento para transmitir e trocar saberes e experiências entre profissionais e familiares com isso reduzindo o número de intervenções e diminuindo a frequência de infecções hospitalares - e também para minimizar os traumas ocorridos durante os dias de internação (Andrade, 1993; Barrera et al., 1993).

Outro exemplo dessa temática foi um projeto com mães de crianças hospitalizadas que se realizou em São Paulo na década de 1980, o qual garantia a permanência das mães ou outro cuidador durante as 24 horas do dia, estimulando-os a participarem das práticas assistenciais e fortalecendo o desenvolvimento e a recuperação das crianças (Mora et al., 1991).

No Chile, estudo comprova que, com a permanência das mães na pediatria, há um decréscimo de $20 \%$ no tempo de internação (Barrera et al., 1993). Além disso, quando as mães acompanham seus filhos, é possível que auxiliem os profissionais nos procedimentos de menor complexidade (Escobar, 1998). No entanto, para que isso ocorra, é fundamental que a equipe de saúde esteja disponível a também sofrer mudanças, em especial no que diz respeito ao acolher e auxiliar a família nesse período de modificações e adaptações no ambiente hospitalar (Silveira e Carvalho, 2002).

No âmbito da saúde mental, mesmo a partir da reforma psiquiátrica, observam-se reflexos do período manicomial. Pesquisa revela que a inserção social é um grande desafio para os profissionais, tornando-se necessário romper os paradigmas impostos pela sociedade, para promover a atenção integral a esses sujeitos (Moraes, 2008). Nesse contexto, um estudo salienta que é preciso promover o acolhimento com estratégia clínica, para superar os percalços enfrentados pela pessoa com transtorno psíquico, e substituir o modelo hospitalar vigente pela continuidade da assistência em redes de cuidado em saúde, a qual englobe a comunidade e os ambulatórios (França, 2005).

A criação de grupos é um fator destacado dos estudos como uma alternativa válida a ser adotada pelos serviços de saúde. Dissertações que tiveram gestantes como sujeitos de pesquisa revelam que, ao participarem de grupos, elas sentem-se acolhidas e seguras e conseguem partilhar anseios e inquietações, além de trocar experiências (Kesselring, 2001; Pereira, 2006).

O processo de capacitação profissional também é citado como modelo de organização. A capacitação é vista como fundamental para superar as dificuldades encontradas no cotidiano de trabalho, em razão das insuficiências da estrutura dos serviços no que corresponde ao processo de escuta qualificada, triagem e referenciamento (Leite, Maia e Sena, 1999). Além disso, 
auxilia na superação do modelo cartesiano, o que permite direcionar o comportamento profissional para uma assistência mais humanizada, possibilitando que o período de internação ou enfrentamento de uma patologia se torne menos sofrido (Silva, Sanches e Carvalho, 2007; Cordeiro, 2008).

Nos serviços especializados, a capacitação também é referenciada. No atendimento às gestantes, ajuda nas formulações de orientações e estratégias de cuidado, obtém maior eficácia na assistência e reduz a morbimortalidade materno-infantil (Versiani et al., 2008).

Em estudo realizado em Taiwan, o apoio social nos atendimentos foi diagnosticado como relevante para a organização dos serviços, fazendo com que tanto os gestores como os pacientes demonstrassem maior responsabilização. A autonomia dos sujeitos também ficou em maior evidência (Hsu et al., 2006).

Para a atenção humanizada de qualidade, é necessária a integração dos saberes em todos os níveis de gestão dos serviços de saúde, ou seja, é preciso que as ações sejam alicerçadas em vários núcleos de profissões, a fim de planejar uma assistência compartilhada (Versiani et al. 2008; Backes, Koerich e Erdmann, 2007).

O acolhimento é outro fator destacado. Um estudo caracteriza o acolhimento como uma tecnologia operacional, a qual está em processo de construção, sofre alterações nos diferentes cenários do SUS e não existe nas UBS, em razão dos níveis de concepções e das maneiras de se reorganizar o cotidiano de serviço (Souza, Elizabethe et al., 2008).

Um estudo revela que o acolhimento é uma estratégia essencial para a reorganização dos serviços de saúde, porque ocorre uma alteração do foco da assistência somente voltado para o biomédico; ele possibilita o trabalho multiprofissional, o acesso igualitário e maior resolutividade das necessidades de saúde, além de se conciliar com as práticas humanizadas (Silva e Alves, 2008). Além disso, há evidências de que nas unidades de ESF que desenvolvem o acolhimento existe uma adesão maior ao tratamento em razão da criação de vínculo (Sá et al., 2007).

As tecnologias das relações, do mesmo modo, são consideradas como alternativas para modificar a organização no serviço (Schimith, 2002; Martins e Nascimento, 2005; Gama et al., 2009). Na maior parte dos achados, esse tipo de tecnologia é mais utilizado pela equipe de enfermagem (Rossi e Lima, 2005).

Uma pesquisa realizada nos EUA identificou um considerável grau de satisfação dos pacientes quando, durante o atendimento, apareciam nos profissionais da saúde os sentimentos de compreensão, honestidade e confiança (Stock Keister et al., 2004).

Cabe ainda destacar um estudo que revela a diferenciação do modo de organização dos serviços nas instituições públicas e nas privadas. Essas variações englobam a diferença da assistência e o processo de relações entre os 
profissionais e usuários, a conduta em relação à dor durante o trabalho de parto, as condições de continuidade das consultas de pré-natal e as relações de confiança entre os médicos e os pacientes (Gama et al., 2009).

\section{(Des)colonialismo}

Neste núcleo de sentido, foram agrupados os resultados que indicam a existência de práticas, concepções e relações opressoras na sociedade e nos serviços de saúde ou, ao contrário, práticas, concepções e relações solidárias.

Destaca-se a área de saúde da mulher. Um estudo brasileiro, de Alves e Brandão (2009), revelou que o relacionamento entre adolescentes apresenta forte hierarquia de gênero. Outro, da República Tcheca, identificou mulheres idosas e com menos escolaridade sendo mais submissas nas atitudes do que homens jovens e com mais educação, estes tendo atitudes autônomas (Krizova e Simek, 2007). A crítica às relações de gênero é feita ao modelo de assistência ao parto, ao qual se submete quem deve ser sujeito, inviabilizando um lugar de poder diferenciado para as usuárias (Gama et al., 2009).

Um estudo envolvendo a mulher demonstrou a necessidade de reconstrução de práticas que fortaleçam a autonomia e a autoconfiança das mulheres (Souza, Kleyde et al., 2008). Uma pesquisa revelou que a episiotomia é realizada sem informação e sem autorização prévia das mulheres, revelando a posição de autoridade dos profissionais durante o parto, destituindo a mulher de poder decisório (Santos e Shimo, 2008). Reforçando esse achado, outra pesquisa verificou que a representação das mulheres entre os profissionais é de mulher-mãe, como vítima, enquanto a representação entre as pacientes é da 'mulher-guerreira', conseguindo negociar com os profissionais suas necessidades, contrariando o mito da passividade (Aguiar e Simões-Barbosa, 2006). Em outra, foi evidenciado que a mulher desconhece os direitos que lhe garantem o serviço especializado em atender mulheres vítimas de violência sexual, atrasando o atendimento e diminuindo a eficácia do atendimento (Oliveira et al., 2005).

Pesquisas revelam que a relação interpessoal entre paciente feminina e profissional foi eminentemente técnica, com a assistência sendo prestada baseada em preconceitos e julgamentos negativos acerca da mulher sob seus cuidados (Motta, 2005) ou culpabilizante (Presser, Meneghel e Hennington, 2008). A violência institucional velada também foi descrita no processo parturitivo, desrespeitando os princípios do Programa de Humanização do Nascimento do Ministério da Saúde (Moura et al., 2007). As demandas trazidas pelas mulheres não foram acolhidas, sendo priorizadas as necessidades biológicas, em detrimento da intersubjetividade e dos projetos de felicidade (Landroni, 2004). Entretanto, outra pesquisa revela que o cuidado oferecido 
pelas enfermeiras obstétricas promove e favorece a emancipação das mulheres usuárias (Quitete e Vargens, 2009).

A humanização no cuidado de enfermagem à mulher parturiente e família está condicionada à valorização da intersubjetividade, ao 'estar com' e 'fazer com', ao predomínio da relação Eu-Tu em detrimento da relação Eu-Isso (Santos, 1998), ou seja, ser alguém com direito a diferença (Souza, Kleyde et al., 2008). A qualificação do atendimento no pré-natal e no parto também passa pela necessidade de reconhecimento, por parte dos profissionais, médicos e demais profissionais da saúde, das necessidades das usuárias e não das conveniências dos profissionais e de suas corporações, respeitando as mulheres como pessoas, com direitos humanos e reprodutivos (Rocha, 2004).

A saúde da criança foi destaque também nesse núcleo de sentido. Está relacionada com a permanência de pais e familiares nas unidades de internação e com o cuidado ao neonato prematuro. Indica que os profissionais não medeiam suas ações com base em uma efetiva relação social profissionalfamília-criança (Franco e Aguillar, 2007), ou que entendem que a humanização do cuidar pressupõe o compartilhar de perspectivas e cuidados (Gomes e Erdmann, 2005). Levar a mãe para a enfermaria, compartilhar saberes, poderes e espaço não se constituem em atos simples, implicam mudanças de valores e atitudes dos profissionais e pais (Lima, Rocha e Scochi, 1999).

Estudo realizado com profissionais que atuam em UTI pediátrica revelou a família vista como intrusa, atrapalhando e fiscalizando a equipe (Barbosa, 2004). Outro, que investigou a comunicação entre mãe-criança-auxiliares de enfermagem, identificou as categorias bloqueadoras como desaprovação, depreciação, tranquilizando a mãe com comentários estereotipados, sendo as que predominaram (Oliveira e Cassiani, 1997).

Uma dissertação apontou que os profissionais deixam de acolher as famílias não colaborativas, como estratégia de enfrentamento à sobrecarga de trabalho e também pela indefinição do papel do acompanhante de adultos ou idosos (Dell' Acqua, 2008).

Os enfermeiros vivenciam a autonomia do usuário/família de forma conflitante: por um lado, respeitam; por outro, consideram necessário impor limites para os usuários do serviço (Moraes et al., 2009).

Os usuários valorizam mais o profissional afetivo e que os inclui nas decisões clínicas, e criticam a atitude distante, fria e bruta, comparando-a à de um 'cavalo batizado' (Nations e Gomes, 2007). Nesse sentido, Kalakum, Viegas e Gerhardt (1995) revelam a importância da participação do sujeito na tomada de decisão sobre seu tratamento; e Hayashi e Gisi (2000), a necessidade de se encontrarem soluções para os problemas dos usuários e de se desenvolver a capacidade de tomada de decisão consciente.

A tomada de decisão compartilhada é foco de várias pesquisas internacionais. Uma delas destaca os elementos necessários para capacitar o paciente 
para participar na tomada de decisão (Fraenkel e McGraw, 2007). Entre os cidadãos lituanenses, as pessoas jovens e com mais educação confiam menos no sistema de saúde, mas são mais motivadas a participar ativamente na tomada de decisão no cuidado em saúde (Grabauskas Peicius e Kaminskas, 2004). Entretanto, pesquisa com pacientes diabéticos indicou que a comunicação efetiva com os profissionais é mais importante do que a participação na tomada de decisão (Heisler et al., 2002).

Pesquisa com o objetivo de caracterizar a natureza e a abrangência da tomada de decisão informada em consultas de rotina com médicos de atenção primária e cirurgiões verificou que a informação e a tomada de decisão foram, com frequência, incompletas, mesmo para decisões de menor complexidade, concluindo que é necessário encorajar decisões informadas na prática clínica (Braddock et al., 1999).

Assim, o usuário é tido, por vezes, como cidadão, e por vezes como um objeto (Schimith, 2002). Em um ambulatório de especialidades médicas, alguns profissionais imputam culpa ao doente, tachando-o de malandro e desinteressado no cuidado com sua saúde (Campinas, 2004). Em um hospital, os pacientes não sabem qual patologia os afeta, por consequência, ao ser negado o direito ao conhecimento sobre seu próprio organismo e à autonomia de cuidar de si, é também negado o direito de cidadania (Rocha, 2007). Destacase o papel do enfermeiro no planejamento do cuidado ao paciente e sua família (Kalakum, Viegas e Gerhardt, 1995). No entanto, a complexidade da saúde, considerando que deva ser pensada no itinerário de vida de cada sujeito, pressupõe diálogo, pertencendo ao âmbito comunitário em seu conjunto, não admitindo exclusividade de especialistas (Najmanovich e Lennie, 2004).

\section{Discussão}

A temática pesquisada é ampla e apresenta um considerável número de publicações. No entanto, percebe-se, pelo recorte temporal, que é recente a preocupação dos pesquisadores em abordá-la, mesmo em pesquisas nacionais, considerando a implantação do SUS. A produção do Brasil foi maior quando comparada à internacional. Isso pode ser creditado à Política Nacional de Humanização, existente desde 2003, que preconiza trocas solidárias entre gestores, trabalhadores e usuários (Brasil, 2004). A enfermagem, destaque nas publicações analisadas, apresentou um movimento de internacionalização das produções científicas a partir de 2000, com o estabelecimento de intercâmbios da pós-graduação brasileira com universidades estrangeiras (Salles e Barreira, 2010). Portanto, também é um elemento que justifica esse aumento recente.

Historicamente, no Brasil, a Saúde da Mulher e a Saúde da Criança foram priorizadas nas políticas públicas de saúde. A saúde mental contribuiu com 
a diferenciação de cenários, uma vez que a reforma psiquiátrica prevê a desinstitucionalização com a substituição progressiva dos manicômios e o estímulo à construção de rede de cuidados interligando Caps, ESF e UBS, portanto, novos itinerários, novos circuitos de saúde, na esperança de lugares mais condizentes com a cidadania do sujeito com sofrimento psíquico (Dalmolin, 2006).

No entanto, nos resultados do cenário global da pesquisa, essa situação se inverte, aparecendo a área hospitalar com maior produção nessa temática. Se por um lado isso revela que a produção científica na temática está centrada no hospital, por outro desvenda o movimento desse espaço para se tornar mais humano, menos biomédico. No Brasil, onde os estudos se concentraram, o Ministério da Saúde lançou em 2001 o Programa Nacional de Humanização da Assistência Hospitalar (PNHAH), que pode explicar esse aumento.

Os núcleos de sentido apresentam-se entrecruzados, de modo que a discussão será realizada em conjunto. A principal tônica dos resultados indica a urgência de se tomar o usuário dos serviços de saúde como sujeito com direito de cidadania, digno de respeito e singularização.

A importância do papel da interação com o profissional de saúde, em especial o enfermeiro, para além da atuação técnica foi destaque nos resultados. Está associada à criação e manutenção de vínculo, à adesão ao plano de cuidados, à satisfação dos usuários. A isso se pode designar de coprodução de problematização, sendo imprescindível o reconhecimento por parte da equipe do poder das pessoas para interferirem na relação com a sua própria vida e o adoecimento (Oliveira, 2007).

Chamam a atenção os distintos caminhos percorridos pelos pesquisadores e trabalhadores das áreas da mulher e da criança. O mote principal das pesquisas e experiências da saúde da criança está dirigido à permanência dos pais nas unidades de internação hospitalares, e muitas delas revelam a necessidade de controle ainda vigente nos serviços de saúde. A saúde da mulher, ao contrário, apresenta uma diretriz marcada pelo direito de cidadania, pela luta contra a opressão, imprimindo um movimento contrahegemônico numa sociedade sexista. Esse achado evidencia padrão ético dos pesquisadores, ao encontro da sociologia feminista que desempenhou o papel de produzir a melhor teoria crítica nas últimas décadas (Santos, 2009).

A enfermagem desponta em pesquisas que têm como objetivo a percepção dos sentimentos e significados para usuários e profissionais acerca do adoecimento ou de ações em saúde em diversos cenários do cuidado. Identificou-se que a pesquisa na enfermagem objetiva compreender o seu objeto de trabalho e melhorar o atendimento ao usuário. No entanto, permanecem lacunas importantes na avaliação das ações desenvolvidas, sendo necessário ampliar o olhar da enfermagem na gestão do cuidado e na dimensão 
ética nesse processo, remetendo à incorporação da pesquisa à prática profissional, indo além da objetividade, em busca de um cuidado integral e um viver humano digno (Lunardi et al., 2009).

A opção para analisar esses resultados foi evidenciar a permanência de relações colonialistas nos serviços de saúde, seguindo a proposta de emancipacão dos sujeitos. A inclusão dos usuários nas decisões está atrás apenas da comunicação efetiva com os profissionais, que é condição para o diálogo. O acesso aos serviços de saúde e a preferência por um profissional de saúde que enxergue o usuário como sujeito, não somente como um corpo, também são sinalizados. O vínculo e a tomada de decisões compartilhadas, via de regra, são fundamentais para a adesão e a continuidade do tratamento. O colonialismo é considerado "a incapacidade de reconhecer o outro como igual, a objetivação do outro - transformar o outro em objeto" (Santos, 2007, p. 53). A questão é: há hierarquia nos conhecimentos dos profissonais e usuários? Para construir a emancipação, é preciso "uma nova relação entre o respeito à igualdade e o princípio do reconhecimento da diferença" (Santos, 2007, p. 62, grifo do autor). Descolonizar nossas mentes é necessário para que possamos distinguir as hierarquias, levando em consideração as diferenças que ficarem depois disso (Santos, 2007). Reconhecer as diferenças pressupõe a luta pela igualdade, "porque o importante não é a homogeneização, mas as diferenças iguais" (Santos, 2007, p. 63).

\section{Considerações finais}

A reflexão sobre o material teórico analisado nos dá segurança para afirmar que por mais que o SUS esteja ancorado em leis com status de políticas de Estado, a sua concretude também depende das relações estabelecidas entre os profissionais e usuários da saúde.

Esta pesquisa integrativa, além de permitir a sistematização da produção do conhecimento, possibilitou identificar potencialidades e fragilidades nesse campo do saber, e quiçá poderá estimular a construção de novos estudos. As potencialidades identificadas apontam para a dimensão transformadora das práticas em saúde quando se considera o usuário como sujeito no processo. Já as fragilidades evidenciam a permanência da visão biologista e fragmentada nos serviços. No entanto, este artigo apresenta limites, em razão da ampla publicação da temática; foram analisados somente os resumos das produções científicas, dificultando assim o aprofundamento dos dados coletados. 


\section{Colaboradores}

Maria Denise Schimith e Bruna Sodré Simon trabalharam em conjunto no desenvolvimento desta pesquisa e em todas as etapas de produção do manuscrito. Ana Cristina Passarella Brêtas e Maria de Lourdes Denardin Budó participaram na elaboração deste artigo.

\section{Notas}

1 Professora assistente do Departamento de Enfermagem da Universidade Federal de Santa Maria (UFSM), Santa Maria, Rio Grande do Sul, Brasil. Doutoranda em Ciências da Saúde pelo Doutorado Interinstitucional Novas Fronteiras Universidade Federal de São Paulo/UFSM. <ma.denise2011@gmail.com>

Correspondência: Rua João Goulart, 519, CEP 97105-220, Camobi, Santa Maria, Rio Grande do Sul, Brasil.

2 Bolsista do Programa Especial de Incentivo à Pesquisa para o Servidor Mestre (PEIPSM). Acadêmica do curso de Enfermagem da Universidade Federal de Santa Maria (UFSM), Santa Maria, Rio Grande do Sul, Brasil. <bru.simon@hotmail.com>

3 Professora associada da Escola Paulista de Enfermagem da Universidade Federal de São Paulo (Unifesp), São Paulo, SP, Brasil. Doutora em Enfermagem pela Unifesp. <acpbretas@unifesp.br>.

4 Professora associada do Departamento de Enfermagem e do Programa de Pós-Graduação em Enfermagem da Universidade Federal de Santa Maria (UFSM), Santa Maria, Rio Grande do Sul, Brasil. Doutora em Enfermagem pela Universidade Federal de Santa Catarina. $<$ lourdesdenardin@gmail.com>

5 Projeto de pesquisa financiado pelo Programa Especial de Incentivo à Pesquisa para o Servidor Mestre (PEIPSM) da Universidade Federal de Santa Maria. 


\section{Referências}

ABDALLA, Fermanda Tavares de Mello; NICHIATA, Lúcia Yasuko Izumi. A abertura da privacidade e o sigilo das informações sobre o HIV/Aids das mulheres atendidas pelo Programa Saúde da Família no município de São Paulo, Brasil. Saúde e Sociedade, São Paulo, v. 17, n. 2, p. 140-152, abr./jun.2008.

AGUIAR, Janaína Marques; SIMÕES-BARBOSA, Regina Helena. Relações entre profissionais de saúde e mulheres HIV+: uma abordagem de gênero. Cadernos de Saúde Pública, Rio de Janeiro, v. 22, n. 10, p. 2.115-2.123, out.2006.

ALMEIDA, Mariza Silva; SILVA, Isília Aparecida. Necessidades de mulheres no puerpério imediato em uma maternidade pública de Salvador, Bahia, Brasil. Revista da Escola de Enfermagem da USP, São Paulo, v. 42, n. 2, p. 347-354, jun.2008.

ALVES, Camila Aloísio; BRANDÃo, Elaine Reis. Vulnerabilidades no uso de métodos contraceptivos entre adolescentes e jovens: interseções entre políticas públicas e atenção à saúde. Ciência \& Saúde Coletiva, Rio de Janeiro, v. 14, n. 2, p. 661-670, mar./abr.2009.

AMESTOY, Simone Coelho; SCHUWARTZ, Eda; THOFEHRN, Maria Buss. A humanização do trabalho para os profissionais de enfermagem. Acta Paulista de Enfermagem, São Paulo, v. 19, n. 4, p. 444-449, out./dez.2006.

ANDRADE, Vera Rodrigues Oliveira. Interação criança/mãe/equipe de enfermagem em processo de hospitalização. Revista Enfermagem Uerj, Rio de Janeiro, v. 1, n. 1, p. 28-35, jun.1993.

ARAÚJO, Anelize Teresinha da Silva; LOBO, Lilia Ferreira. Além do rio psicoterapêutico: reflexões atuais sobre uma experiência de acolhimento e inclusão. Psico- logia Argumento, Curitiba, v. 25, n. 51, p. 361-370, out.2007.

ARAÚJO, Bárbara Bertolossi Marta; RODRIGUES, Benedita Maria Rêgo Deusdará; RODRIGUES, Elisa da Conceição. O diálogo entre a equipe de saúde e mães de bebês prematuros: uma análise freireana. Revista Enfermagem Uerj, Rio de Janeiro, v. 16, n. 2, p. 180-186, abr./jun.2008.

ASSUNÇÃO, Thaís Silva; URSINE, Priscila Guedes Santana. Estudo de fatores associados à adesão ao tratamento não farmacológico em portadores de diabetes mellitus assistidos pelo Programa Saúde da Família, Ventosa, Belo Horizonte. Ciência \& Saúde Coletiva, Rio de Janeiro, v. 13, n. 2, p. 2.189-2.197, dez.2008.

BACKES, Dirce Stein; KOERICH, Magda Santos; ERDMANN, Alacoque Lorenzini. Humanizing care through the valuation of the human being: resignification of values and principles by health professionals. Revista Latino-Americana de Enfermagem, Ribeirão Preto, v. 15, n. 1, p. 34-41, jan./fev. 2007.

BACKES, Dirce Stein; LUNARDI FILHO, Wilson Danilo; LUNARDI, Valéria Lerch. A construção de um ambiente de cuidado humano: percepção dos integrantes do grupo de humanização. Nursing, Barueri, v. 9, n. 101, p. 1.057-1.063, out.2006.

BARBOSA, Elizabeth Carla Vasconcelos. Humanização nas relações com a família: um desafio para a enfermagem em UTI pediátrica. Acta Scientiarum: Ciências da Saúde, Maringá, v. 26, n. 1, p. 205-212, jan./jun.2004.

BARRA, Daniela Couto Carvalho et al. Processo de humanização e a tecnologia para o paciente internado em uma unidade de terapia intensiva. Reme - Revista Mineira de Enfermagem, Belo Horizonte, v. 9, n. 4, p. 341-347, out./dez.2005. 
BARRERA, Francisco et al. Participación materna en la atención del niño hospitalizado. Revista Chilena de Pediatria, Santiago, v. 64, n. 3, p. 164-168, mayo/jun.1993.

BECK, Carmem Lúcia et al. Humanização da assistência de enfermagem: percepção de enfermeiros nos serviços de saúde de um município. Revista Gaúcha de Enfermagem, Porto Alegre, v. 30, n. 1, p. 54-61, mar.2009.

BERRY, Leonard et al. Patients' commitment to their primary physician and why it matters. Annals of Family Medicine, Leawood, v. 6, n. 1, p. 6-13, jan./fev.2008.

BICCA, Larissa Heckler; TAVARES, Kátia Ott. A atuação da enfermeira no Programa Saúde da Família: uma breve análise da sua prática assistencial. Nursing, Barueri, v. 9, n. 92 , p. 632-637, jan.2006.

BORSOI, Tatiana dos Santos; BRANDÃO, Elaine Reis; CAVALCANTI, Maria de Lourdes Tavares. Ações para o enfrentamento da violência contra a mulher em duas unidades de atenção primária à saúde no município do Rio de Janeiro. Interface - Comunicação, Saúde, Educação, Botucatu, v. 13, n. 28, p. 165-174, jan./mar.2009.

BRADDOCK, Clarence et al. Informed decision making in outpatient practice: time to get back to basics. Journal American Medical Association, Chicago, v. 282, n. 24, p. 2.3132.320, Dec.1999.

BRASIL. Ministério da Saúde. SecretariaExecutiva. Núcleo Técnico da Política Nacional de Humanização. HumanizaSUS: Política Nacional de Humanização: a humanização como eixo norteador das práticas de atenção e gestão em todas as instâncias do SUS - Ministério da Saúde, Secretaria-Executiva, Núcleo Técnico da Política Nacional de Humanização. Brasília: Ministério da Saúde, 2004.

BRAVO, Paulina; URIBET, Claudia; CONTRERAS, Aixa. El cuidado percibido durante cel proceso de parto: una mirada desde las madres. Revista Chilena de Obstetricia y
Ginecologia, Santiago, v. 73, n. 3, p. 179184, 2008.

BRONFMAN-PERTZOVSKY, Mario Norberto; LÓPEZ-MORENO, Sergio; MAGIS-RODRÍGUEZ, Carlos; MORENO-ALTAMIRANO, Alejandra; RUTSTEIN, Shea. Atención prenatal en el primer nivel de atención: características de los proveedores que influyen en la satisfacción de las usuarias. Salud pública de México; Cuernavaca, v. 45, n. 6, p. 445-454, nov.-dic. 2003. ilus.

CAETANO, Joselany Afio et al. Cuidado humanizado em terapia intensiva: um estudo reflexivo. Escola Anna Nery Revista de Enfermagem, Rio de Janeiro, v. 11, n. 2, p. 325-330, jun. 2007.

CAMPINAS, Lúcia de Lourdes Souza Leite. O acolhimento ao doente com tuberculose: estudo comparativo entre uma unidade de saúde da família e um ambulatório de especialidades médicas, São Paulo/SP, 2003. Tese (Doutorado em Saúde Pública) - Faculdade de Saúde Pública, USP, São Paulo, 2004.

CARRET, Maria Laura Vidal; FASSA, Ana Claudia Gastal; DOMINGUES, Marlos Rodrigues. Prevalência e fatores associados ao uso inadequado do serviço de emergência: uma revisão sistemática da literatura. Cadernos de Saúde Pública, Rio de Janeiro, v. 25, n. 1, p. 7-28, jan.2009.

COELHO, Márcia Oliveira; JORGE, Maria Salete Bessa. Tecnologia das relações como dispositivo do atendimento humanizado na atenção básica à saúde na perspectiva do acesso, do acolhimento e do vínculo. Ciência \& Saúde Coletiva, Rio de Janeiro, v. 14, supl. 1, p. 1.523-1.531, set./out.2009.

CORDEIRO, Tania Maria de Oliviera. Avaliação da organização da assistência às doenças sexualmente transmissíveis na rede básica de saúde do município de Ilhéus, BA. Dissertação (Mestrado em Avaliação em Saúde) - Escola Nacional de Saúde Pública Sergio Arouca/Fiocruz, Rio de Janeiro, 2008. 
DALMOLIN, Bernadete Maria. Esperança equilibrista: cartografias de sujeitos em sofrimento psíquico. Rio de Janeiro: Editora Fiocruz, 2006.

DAVIM, Rejane Marie Barbosa et al. Opinião de puérperas quanto ao acolhimento no alojamento conjunto de uma maternidade pública de Natal-RN. Nursing, Barueri, v. 12, n. 137, p. 479-484, out.2009.

DELFINI, Patrícia Santos de Souza; SATO, Miki Takao; ANTONELI, Patrícia de Paulo. Parceria entre Caps e PSF: o desafio da construção de um novo saber. Ciência \& Saúde Coletiva, Rio de Janeiro, v. 14, supl. 1, p. 1.4831.492, set./out.2009.

DELL'ACQUA, Marcos Lourenço. Do acolhimento à desconsideração da família no cenário hospitalar pela enfermagem: a divergência de modelos assistenciais e o sofrimento no trabalho, como componentes intervenientes. Dissertação (Mestrado em Enfermagem) - Faculdade de Medicina de Botucatu, UEP, Botucatu, SP, 2008.

DEWAR, Anne et al. Navigating the health care system: perceptions of patients with chronic pain. Chronic Diseases Canada, Ottawa, v. 29, n. 4, p. 162-168, 2009.

DUARTE, Sebastião Junior Henrique; ANDRADE, Sônia Maria Oliveira de. Assistência pré-natal no Programa Saúde da Família. Escola Anna Nery Revista de Enfermagem, Rio de Janeiro, v. 10, n. 1, p. 121126, abr.2006.

ESCOBAR, Sonia. Participación materna en la atención del niño hospitalizado. Revista Chilena de Pediatria, Santiago, v. 14, n. 3, p. 158-60, jul./ago.1998.

FAIMAN, Carla Segre et al. Os cuidadores: a prática clínica dos profissionais da saúde. Mundo Saúde, Vitória, v. 27, n. 2, p. 254257, abr./jun.2003.

FAQUINELLO, Paula; HIGRASHI, Ieda Harumi; MARCON, Sonia Silva. O atendi- mento humanizado em unidade pediátrica: percepção do acompanhante da criança hospitalizada. Texto \& Contexto Enfermagem, Florianópolis, v. 16, n. 4, p. 609-616, out./dez.2007.

FRAENKEL, Raenkel Liana; McGRAW, Sarah. What are the essential elements to enable patient participation in medical decision making? Journal of General Internal Medicine, Philadelphia, v. 22, n. 5, p. 614-619, May 2007.

FRANÇA, Irvalete Guimarães. Reflexões acerca da implantação e funcionamento de um plantão de emergência em saúde mental. Psicologia: Ciência e Profissão, Brasília, v. 25, n. 1, p. 146-163, 2005.

FRANCO, Sonia Maria Carneiro de Morais; AGUILLAR, Olga Maimoni. Criança hospitalizada: compreendendo as necessidades de mães durante a hospitalização. Nursing, Barueri, v. 9, n. 107, p. 166-170, abr.2007.

GAMA, Andréa de Sousa et al. Representações e experiências das mulheres sobre a assistência ao parto vaginal e cesárea em maternidades pública e privada. Cadernos de Saúde Pública, Rio de Janeiro, v. 25, n. 11, p. $2.480-2.488$, nov. 2009.

GANZELLA, Marcela; ZAGO, Márcia Maria Fontão. A alta hospitalar na avaliação de pacientes e cuidadores: uma revisão integrativa da literatura. Acta Paulista de Enfermagem, São Paulo, v. 21, n. 2, p. 351355, dez.2008.

GOMES, Anna Luiza Castro; SÁ, Lenilde Duarte. As concepções de vínculo e a relação com o controle da tuberculose. Revista da Escola de Enfermagem da USP, São Paulo, v. 43, n. 2, p. 365-372, jun.2009.

GOMES, Giovana Calcagno; ERDMANN, Alacoque Lorenzini. O cuidado compartilhado entre a família e a enfermagem à criança no hospital: uma perspectiva para a sua humanização. Revista Gaúcha de Enfermagem, Porto Alegre, v. 26, n. 1, p. 20-30, abr.2005. 
GOMES, Lucy; VIANNA, Cecília. Interação do profissional de saúde com o paciente idoso. Jornal Brasileiro de Medicina, Rio de Janeiro, v. 95 , n. 5/6, p. 36-39, nov./dez.2008.

GOTARDO, Glória Inês Beal; SILVA, Claudia Aparecida. O cuidado dispensado aos familiares na unidade de terapia intensiva. Revista Enfermagem Uerj, Rio de Janeiro, v. 13, n. 2, p. 223-228, maio/ago. 2005.

GRABAUSKAS VILIUS, Peicius Eimantas; KAMINSKAS, Raimundas. The patient role in decision-making in Lithuanian health care. Medicina (Kaunas), Kaunas, v. 40, n. 11, p. 1.109-1.116, 2004.

HAYASHI, Alda Aparecida Mastelaro; GISI, Maria Lourdes. O cuidado de enfermagem no CTI: da ação-reflexão à conscientização. Texto \& Contexto Enfermagem, Florianópolis, v. 9 , n. 2(pt.2), p. 824-837, maio/ago.2000.

HEISLER, Michele et al. The relative importance of physician communication, participatory decision making, and patient understanding in diabetes self-management. Journal of General Internal Medicine. Philadelphia, v. 17, n. 4, p. 243-252, Apr. 2002.

HOYOS, Hernández Paula Andrea; CARDONA, Ramírez Mónica Alexandra; CORREA, Sánchez Diego. Humanizar los contextos de salud, cuestión de liderazgo. Investigación y Educación en Enfermería. Medellín, v. 26, n. 2, p. 218-225, Sept. 2008.

HSU, Chih-Cheng et al. The dimensions of responsiveness of a health system: a Taiwanese perspective. BMC Public Health, Londres, v. 6, n. 72, Mar. 2006.

JORGE, Maria Salete Bessa et al. Avaliação da qualidade do Programa Saúde da Família no Ceará: a satisfação dos usuários. Revista Baiana de Saúde Pública, Salvador, v. 31, n. 2, p. 256-266, jul./dez.2007.

KAHOLOKULA, Joseph Keawe'aimoku et al. Pacific Islanders' perspectives on heart failure management. Patient Education and Counseling, Amsterdã, v. 70, n. 2, p. 281-291, Feb. 2008.
KALAKUM, Luciane; VIEGAS, Maria Alice Vargas; GERHARDT, Luiza Maria. A ética, o cliente com câncer e o enfermeiro. Texto \& Contexto Enfermagem, Florianópolis, v. 4, n. 2, p. 38-47, jul./dez. 1995.

KESSELRING, Beatriz Basile de Castro. Preparando-se para enfrentar o parto e pós-parto: a experiência da participação em grupo de gestantes. Dissertação (Mestrado em Enfermagem) - Escola de Enfermagem, USP, São Paulo, 2001.

KRIZOVA, Eva; SIMEK, Jiri. Theory and practice of informed consent in the Czech Republic. Journal of Medical Ethics, Londres, v. 33, n. 5, p. 273-277, May 2007.

LANDRONI, Maria Angela Silva. Aids e gravidez: desafios para o cuidado nos serviços de saúde. Dissertação (Mestrado em Saúde Pública) - Faculdade de Saúde Pública, USP, São Paulo, 2004

LEITE, Juliana; MAIA, Carmem Araújo; SENA, Roseni Rosângela. Acolhimento: perspectiva de reorganização da assistência de enfermagem. Revista Brasileira de Enfermagem, Brasília, v. 52, n. 2, p. 161-168, abr./jun.1999.

LIMA, Luciana Bjorklund; BUSIN, Lurdes. O cuidado humanizado sob a perspectiva de enfermeiras em unidade de recuperação pós-anestésica. Revista Gaúcha de Enfermagem, Porto Alegre, v. 29, n. 1, p. 90-97, mar.2008.

LIMA, Maria da Glória. Assistência prestada pelo enfermeiro em unidades de terapia intensiva: aspectos afetivos e relacionais. Dissertação (Mestrado em Enfermagem) Escola de Enfermagem de Ribeirão Preto, USP, Ribeirão Preto, SP, 1993.

LIMA, Regina Aparecida Garcia; ROCHA, Semiramis Melani Melo; SCOCHI, Carmen Gracinda Silvan. Assistência à criança hospitalizada: reflexões acerca da participação dos pais. Revista Latino-Americana de Enfermagem, Ribeirão Preto, SP, v. 7, n. 2, p. 33-39, abr.1999. 
LUNARDI, Valéria Lerch et al. Impacto dos resultados das pesquisas em enfermagem na prática profissional. Cogitare Enfermagem, Curitiba, PR, v. 14, n. 1, p. 165-171, jan./ mar.2009.

MAGYAR-RUSSELL, Gina et al. Ophthalmology patients' religious and spiritual beliefs: an opportunity to build trust in the patient-physician relationship. Archives of Ophthalmology, Chicago, v. 126, n. 9, p. 1.262-1.265, sep.2008.

MARTÍNEZ, Cláudio et al. Alianza terapéutica en un grupo de pacientes de nivel socio-económico bajo. Revista Argentina Clínica Psicologica, Buenos Aires, v. 6, n. 2, p. 123-133, ago.1997.

MARTINS, Josiane de Jesus; NASCIMENTO, Eliane Regina Pereira. Repensando a tecnologia para o cuidado do idoso em UTI. ACM - Arquivos Catarinenses de Medicina, Florianópolis, v. 34, n. 2, p. 49-55, abr./ jun.2005.

MATSUDA, Laura Misue; SILVA, Neuza; TISOLIN, Ana Maria. Humanização da assistência de enfermagem: estudo com clientes no período pós-internação de uma UTIadulto. Acta Scientiarum: Health Science, Maringá, v. 25, n. 2, p. 163-170, jul./dez.2003.

MATTOS, Ruben Araujo. Integralidade, trabalho, saúde e formação profissional: algumas reflexões críticas feitas com base na defesa de alguns valores. In: MATTA, Gustavo Corrêa; LIMA, Julio César França (Orgs.). Estado, sociedade e formação profissional: contribuições e desafios em 20 anos de SUS. Rio de Janeiro: Editora Fiocruz, 2008. p. 313-352.

MENDES, Karina Dal Sasso; SILVEIRA, Renata Cristina de Campos Pereira; GALVÃO, Cristina Maria. Revisão integrativa: método de pesquisa para a incorporação de evidências na saúde e na enfermagem. Texto Contexto Enfermagem, Florianópolis, v. 17, n. 4, p. 758-764, out./dez.2008.
MINAYO, Maria Cecília de Souza. O desafio do conhecimento: pesquisa qualitativa em saúde. 11. ed. São Paulo: Hucitec, 2008.

MOLINA, Rosemeire Cristina Moretto et al. A percepção da família sobre sua presença em uma Unidade de Terapia Intensiva Pediátrica e Neonatal. Revista Escola Enfermagem USP, São Paulo, v. 43, n. 3, p. 630638, set.2009.

MORA, Maria Cristina Surani et al. Programa Mãe Participante: experiência de 1 ano de implantação no Hospital Humberto Primo (HUI). Revista Paulista de Pediatria, São Paulo, v. 9, n. 32, p. 14-2l, jan./mar.1991.

MORAES, Edvaldo Leal et al. Vivenciando a autonomia do cliente e da família: percepção de enfermeiros da unidade de terapia intensiva de um hospital escola. Nursing, Barueri, v. 12, n. 135, p. 375-380, ago. 2009.

MORAES, Maristela. O modelo de atenção integral à saúde para tratamento de problemas decorrentes do uso de álcool e outras drogas: percepções de usuários, acompanhantes e profissionais. Ciência \& Saúde Coletiva, Rio de Janeiro, v. 13, n. 1, p. 121133, jan./fev. 2008. tab.

MOREIRA, Thereza Maria Magalhães; ARAÚJO, Thelma Leite. Sistema interpessoal de Imogene King: as relações entre pacientes com hipertensão não aderentes ao tratamento e profissionais de saúde. Acta Paulista de Enfermagem, São Paulo, v. 15, n. 3, p. 34-43, jul./set.2002.

MOTTA, Ilse Sodré. A relação interpessoal entre profissionais de saúde e a mulher em abortamento incompleto: "o olhar da mulher". Revista Brasileira de Saúde Materno Infantil, Recife, v. 5, n. 2, p. 219-227, abr./jun.2005.

MOURA, Fernanda Maria de Jesus Pires et al. Humanization and nursing assistance to normal childbirth. Revista Brasileira de Enfermagem, Brasília, v. 60, n. 4, p. 452-455, jul./ago.2007. 
NAJMANOVICH, Denise; LENNIE, Vera. Pasos hacia un pensamiento complejo en salud. Claves en Psicoanálisis y Medicina, Buenos Aires, v. 13, n. 21, p. 70-76, 2004.

NASCIMENTO, Elaine Ferreira; GOMES, Romeu. Iniciação sexual masculina: conversas íntimas para fóruns privados. Ciência \& Saúde Coletiva, Rio de Janeiro, v. 14, n. 4, p. 1.101-1.110, jul./ago.2009.

NASCIMENTO, Paula Thais Avila; TESSER, Charles Dalcanale; POLI NETO, Paulo. Implantação do acolhimento em uma unidade local de saúde de Florianópolis. ACM - Arquivos Catarinenses de Medicina, Florianópolis, v. 37, n. 4, p. 32-34, set./dez. 2008.

NATIONS, Marilyn; GOMES, Annatália Meneses de Amorim. Cuidado, "cavalo batizado" e crítica da conduta profissional pelo paciente-cidadão hospitalizado no Nordeste brasileiro. Cadernos de Saúde Pública, Rio de Janeiro, v. 23, n. 9, p. 2.103-2.112, set.2007.

NERY, Sônia Regina et al. Acolhimento no cotidiano dos auxiliares de enfermagem nas Unidades de Saúde da Família, Londrina (PR). Ciência \& Saúde Coletiva, Rio de Janeiro, v. 14, supl.1, p. 1.411-1.419, set./out.2009.

OLIVEIRA, Adriano et al. A comunicação no contexto do acolhimento em uma unidade de saúde da família de São Carlos, SP. Interface - Comunicação, Saúde, Educação, Botucatu, v. 12, n. 27, p. 749-762, out./ dez.2008.

OLIVEIRA, Eleonora Menicucci et al. Atendimento às mulheres vítimas de violência sexual: um estudo qualitativo. Revista de Saúde Pública, São Paulo, v. 39, n. 3, p. 376382, jun.2005.

OLIVEIRA, Francisco Arsego. Antropologia nos serviços de saúde: integralidade, cultura e comunicação. Interface - Comunicação, Saúde, Educação, Botucatu, v. 6, n. 10, p. 63-74, fev.2002.
OLIVEIRA, Gustavo Nunes. O projeto terapêutico singular. In: CAMPOS, Gastão Wagner de Souza; GUERRERO, André Vinicius Pires (Orgs.). Manual de práticas em Atenção Básica: saúde ampliada e compartilhada. Hucitec: Rio de Janeiro, 2007. p. 283-297.

OLIVEIRA, Iaponira Cortez Costa; COSTA, Solange Fátima Geraldo; SOARES, Maria Julia Guimarães Oliveira. Discurso de médicos e enfermeiros sobre direitos e deveres de gestantes com HIV/Aids no âmbito assistencial: uma abordagem bioética. Nursing, Barueri, v. 10, n. 112, p. 419-424, set.2007.

OLIVEIRA, Viviane Tosta; CASSIANI, Silvia Helena de Bortoli. O processo de comunicação na administração de medicamentos injetáveis em crianças sob a perspectiva da interação entre mãe-criança e auxiliares de enfermagem. Revista Latino-Americana de Enfermagem, Ribeirão Preto, v. 5, n. 4, p. 61-67, out.1997.

PEREIRA, Audrey Vidal. O mundo positivo do HIV a partir da visão dos profissionais de saúde no município de Niterói, RJ. Dissertação (Mestrado em Saúde Pública) - Escola Nacional de Saúde Pública Sergio Arouca, Fundação Oswaldo Cruz (Ensp/Fiocruz), Rio de Janeiro, 2006.

PÉREZ CICILI, Amely et al. La relación médico-paciente en el sistema nacional de salud. Revista Cuba de Medicina General Integral, Ciudad de La Habana, v. 19, n. 6, nov./dic. 2003.

PÉREZ, Claudio et al. Mirada al gineco-obstetra en atención ambulatória. Revista Chilena de Salud Pública, Santiago, v. 7, n. 2, p. 7479, 2003.

PINHEIRO, Roseni; MATTOS, Ruben Araújo. Implicações da integralidade na gestão da saúde. In: PINHEIRO, Roseni; MATTOS, Ruben Araujo (Orgs.). Gestão em redes: práticas de avaliação, formação e participação na saúde. Rio de Janeiro: Cepesc, 2006. p. 11-26. 
POMPEO, Daniele Alcalá; ROSSI, Lídia Aparecida; GALVÃO, Cristina Maria. Revisão integrativa: etapa inicial do processo de validação de diagnóstico de enfermagem. Acta Paulista de Enfermagem, São Paulo, v. 22, n. 4, p. 434-438, 2009.

PRESSER, Adriana Dewes; MENEGHEL, Stela Nazareth; HENNINGTON, Élida Azevedo. Mulheres enfrentando as violências: a voz dos operadores. Saúde e Sociedade, São Paulo, v. 17, n. 3, p. 126-137, jul./set.2008.

QUEIROZ, Maria Veraci Oliveira et al. Indicadores de qualidade da assistência ao nascimento baseados na satisfação de puérperas. Texto \& Contexto Enfermagem, Florianópolis, v. 16, n. 3, p. 479-487, jul./set.2007.

QUITETE, Jane Baptista; VARGENS, Octavio Muniz Costa. O poder no cuidado da enfermeira obstétrica: empoderamento ou submissão das mulheres usuárias? Revista Enfermagem Uerj, Rio de Janeiro, v. 17, n. 3, p. 315-320, jul./set.2009.

ROBERTS, Lisa; BUCKSEY, Sally J. Communicating with patients: what happens in practice? Physical Therapy, Washington, v. 87, n. 5, p. 586-594, Apr./May 2007.

ROCHA, Francisca Auricélia Furtado. A trajetória de gestantes e parturientes pelo Sistema Único de Saúde no Rio de Janeiro. Dissertação (Mestrado em Ciências na Área de Saúde Pública) - Escola Nacional de Saúde Pública Sergio Arouca, Fundação Oswaldo Cruz (Ensp/Fiocruz), Rio de Janeiro, 2004.

ROCHA, Karen Patricía Wilke Ferreira. A educação em saúde no ambiente hospitalar. Nursing, Barueri, v. 9, n. 108, p. 216-221, maio 2007.

RODRIGUES, Márcia Melo Laet; HOGA, Luiza Akiko Komura. Aborto espontâneo e provocado: sentimentos vivenciados pelos homens. Revista Brasileira de Enfermagem, Brasília, v. 59, n. 1, p. 14-19, jan./fev.2006.

ROSSI, Flávia Raquel; LIMA, Maria Alice Dias da Silva. Acolhimento: tecnologia leve nos processos gerenciais do enfermeiro. Revista Brasileira de Enfermagem, Brasília, v. 58, n. 3, p. 305-310, maio/jun. 2005.

ROTER, Debra et al. Interactive and evaluative correlates of dialogue sequence: a simulation study applying the RIAS to turn taking structures. Patient Educaton and Counseling, Amsterdã, v. 71, n. 1, p. 26-33, Apr.2008.

SÁ, Lenilde Duarte et al. Tratamento da tuberculose em unidades de saúde da família: histórias de abandono. Texto \& Contexto Enfermagem, Florianópolis, v. 16, n. 4, p. 712718, out./dez.2007.

SALLES, Eliane Brandão; BARREIRA, Ieda de Alencar. Formação da comunidade científica de enfermagem no Brasil. Texto \& Contexto Enfermagem, Florianópolis, v. 19, n. 1, p. 137-146, jan./mar.2010.

SANTOS, Boaventura de Sousa. Renovar a teoria crítica e reinventar a emancipação social. São Paulo: Boitempo, 2007.

Por que é tão difícil construir uma teoria crítica? In: SANTOS, Boaventura de Sousa. A crítica da razão indolente: contra o desperdício da experiência. 7. ed. São Paulo: Cortez, 2009. p. 23-37.

SANTOS, Jaqueline de Oliveira; SHIMO, Antonieta Keiko Kakuda. Prática rotineira da episiotomia refletindo a desigualdade de poder entre profissionais de saúde e mulheres. Escola Anna Nery Revista de Enfermagem, Rio de Janeiro, v. 12, n. 4, p. 645-650, dez.2008.

SANTOS, Lia Cristina Galvão. Necessidades de familiares da pessoa internada em unidade de terapia intensiva: uma perspectiva compreensiva para a humanização do cuidar. Tese (Doutorado em Enfermagem) Escola de Enfermagem Anna Nery, Rio de Janeiro, 1998.

SCHIMITH, Maria Denise. Acolhimento e vínculo em uma equipe do Programa de Saúde da Família: realidade ou desejo? Dissertação 
(Mestrado em Enfermagem) - Escola de Enfermagem, Universidade Federal do Rio Grande do Sul (UFRGS), Porto Alegre, 2002.

SILVA, Gisele Ferreira; SANCHES, Patrícia Gisele; CARVALHO, Maria Dalva de Barros. Refletindo sobre o cuidado de enfermagem em unidade de terapia intensiva. RemeRevista Mineira de Enfermagem, Belo Horizonte, v. 11, n. 1, p. 94-98, jan./mar.2007.

SILVA, Lívia Gomes; ALVES, Marcelo da Silva. O acolhimento como ferramenta de práticas inclusivas de saúde. Revista de APS, Juiz de Fora, MG, v. 11, n. 1, p. 74-84, jan./mar.2008.

SILVEIRA, Edilene Aparecida Araújo; CARVALHO, Ana Maria Pimenta. Familiares de clientes acometidos pelo HIV/Aids e o atendimento prestado em uma unidade ambulatorial. Revista Latino-Americana de Enfermagem, Ribeirão Preto, SP, v. 10, n. 6, p. 813818, nov./dez.2002.

SOUSA, Ivna Giovana da Silva et al. Prevenção do câncer de colo uterino: percepções de mulheres ao primeiro exame e atitudes profissionais. Revista RENE, Fortaleza, v. 9, n. 2, p. 38-46, abr./jun.2008.

SOUZA, Kleyde Ventura et al. A consulta puerperal: demandas de mulheres na perspectiva das necessidades sociais em saúde. Revista Gaúcha de Enfermagem, Porto Alegre, v. 29, n. 2, p. 175-181, jun.2008.
SOUZA, Elizabethe Cristina Fagundes et al. Acesso e acolhimento na Atenção Básica: uma análise da percepção dos usuários e profissionais de saúde. Cadernos de Saúde Pública, Rio de Janeiro, v. 24, supl. 1, p. 100-110, 2008.

STOCK KEISTER, Mary C. et al. What people want from their family physician. American Family Physician, Kansas City, v. 69, n. 10, p. 2.310, May 2004.

VERSIANI, Clara de Cássia et al. Maternidade segura: relato de experiência. $R e$ vista de APS, Juiz de Fora, MG, v. 11, n. 1, p. 109-114, jan./mar.2008.

VICTOR, Ana Cleide Soares et al. Comunicação verbal de uma equipe médica: percepções e necessidades de visitantes de uma UTI. Acta Scientiarum: Health Science, Maringá, v. 25, n. 2, p. 199-206, jul./dez.2003.

WOISKI, Ruth Oliveira Santos; ROCHA, Daniele Laís Brandalize. Cuidado de enfermagem à criança vítima de violência sexual atendida em unidade de emergência hospitalar. Escola Anna Nery Revista de Enfermagem, Rio de Janeiro, v. 14, n. 1, p. 143150, jan./mar.2010.

YOKAICHIYA, Chizuru Minami et al. Equipes de farmácia e humanização da assistência a pacientes com HIV/Aids: um estudo qualitativo em serviços municipais de São Paulo. Jornal Brasileiro de Aids, São Paulo, v. 7, n. 5, p. 240-247, set./out.2006.

Recebido em 16/03/2011

Aprovado em 29/07/2011 